\title{
Mujeres inmigrantes latinoamericanas: procesos de agencia en contextos de vulnerabilidad
}

\section{Paola Contreras}

Universidad Autónoma de Barcelona paolacontrerashz@gmail.com

Vanessa Alcaide

Universidad de Barcelona valcaide@ub.edu

\section{Resumen}

El presente artículo analiza la agencia de las mujeres latinoamericanas que residen en la ciudad de Barcelona (España). Teóricamente, se trabajó con las aportaciones en torno al concepto de agencia, resistencia y vulnerabilidades. Metodológicamente, se apostó por las historias de vida con el objetivo de reconstruir las experiencias que han influido en las trayectorias del colectivo de estudio. Los resultados evidencian que, a pesar de enfrentar un contexto social, económico y jurídico-normativo adverso, las mujeres inmigrantes muestran una disposición a revertir o transformar dicho escenario con prácticas cotidianas de resistencia, las cuales nos han permitido definir una tipología de agencia.

Palabras clave: migración femenina; agencia; subjetividad; vulnerabilidad; desigualdad

Abstract. Latin American immigrant women: agency processes in contexts of vulnerability

This article analyzes the agency of Latin American women residing in the city of Barcelona (Spain). Theoretically, we worked with the contributions around the concept of agency, resistance, and vulnerabilities. At the methodological level, we opted for life stories to reconstruct the experiences that have influenced the trajectories of the study group. The results show that, despite facing an adverse social, economic, and legal-regulatory context, immigrant women show a willingness to reverse or transform this scenario with daily practices of resistance, which have allowed us to define a typology of agency.

Keywords: female migration; agency; subjectivity; vulnerability; inequality 


\section{Sumario}

\section{Introducción}

2. Debates en torno a la agencia, vulnerabilidades y resistencias

3. Mujeres inmigrantes: una aproximación al contexto
4. Aproximaciones metodológicas

5. Resultados y discusión

6. Conclusiones

Referencias bibliográficas

\section{Introducción}

La producción científica sobre las migraciones internacionales ha proporcionado perspectivas teóricas que han permitido situar, observar y analizar la dimensión, complejidad y heterogeneidad que implica este proceso. Estas contribuciones han evidenciado la feminización que emplaza a las mujeres como protagonistas de los movimientos migratorios transnacionales. De ahí la relevancia teórica y epistemológica que ofrece el concepto de agencia, pues permite reconocer al sujeto femenino más allá de la subalternidad con la cual se ha construido la imagen de las mujeres del «tercer mundo/sur» (Mohanty, 2008). La tendencia a homogenizarlas desde miradas paternalistas que las sitúan como mujeres sumisas y portadoras de valores tradicionales (Güell et al., 2020) no permite ver las respuestas que desarrollan para hacer frente a situaciones que dificultan sus trayectorias vitales y migratorias.

Bajo estas premisas, el presente artículo tiene como objetivo analizar la agencia de las mujeres latinoamericanas que residen en la ciudad de Barcelona. Con ello, buscamos visibilizar la realidad que afrontan en su devenir como inmigrantes. En este sentido, situamos el concepto de agencia como vector de procesos performativos que conllevan transformaciones subjetivas, discursivas y prácticas e instalan mecanismos que tienden a desestabilizar situaciones de vulnerabilidad (Butler, 2014). De este modo, las mujeres latinoamericanas - uno de los colectivos femeninos con mayor presencia tanto en la ciudad de Barcelona como en España- desarrollan estrategias que cuestionan la subalternidad situada poniendo en valor sus aprendizajes y experiencias, pero también evidencian cómo los discursos de integración, los sistemas de protección social o incluso la propia normativa jurídica están envueltos en una retórica que esconde dinámicas de exclusión que limitan sus derechos y oportunidades.

La estructura del artículo se organiza, en primer lugar, en un marco teórico donde profundizamos en torno a la agencia, resistencia y vulnerabilidades, para luego introducir algunas dimensiones del contexto migratorio. En segundo lugar, se presentan los apuntes metodológicos de la investigación, donde proporcionamos detalles de la sistematización y el análisis de la información recopilada de las 25 historias de vida de mujeres latinoamericanas residentes en la ciudad de Barcelona. Finalmente, discutimos sobre las dinámicas migratorias y las respuestas que despliegan las mujeres para enfrentar un contexto adverso. Como resultado del análisis, emerge una tipología de agencia situada desde las experiencias de las participantes. 


\section{Debates en torno a la agencia, vulnerabilidades y resistencias}

Las aportaciones teóricas en torno a la agencia resaltan el rol del sujeto activo y consciente de su lugar de enunciación, que, inmerso en un proceso constante de construcción-deconstrucción de significados (Casado, 2003; Muñoz-Arce, 2018), tiene una disposición subjetiva por transformar el espacio social. En este sentido, la agencia «incluye un poder efectivo para alcanzar las metas establecidas; se relaciona con la capacidad y disposición para actuar en función de los propósitos individuales y colectivos (...), implica responsabilidad en la toma y ejecución de las decisiones» (Domínguez y Contreras, 2017). En concordancia con ello, la capacidad de agencia genera nuevos significados, posibilidades y subjetividades que favorecen abrir espacios de actuación política e individual de los sujetos (Cano, 2017; Salem et al., 2020). Para Sen (1985), la capacidad de agencia se fundamenta en la actuación de cada persona en función de lo que valora, desea o necesita. En última instancia, apunta al bienestar y poder de decisión. Si la agencia individual se centraliza en el poder de decisión, la agencia social lo hace en la capacidad de subvertir las normas (Butler, 2014). No obstante, Mahmood (2008) critica la relación que se establece entre agencia y resistencia a determinadas normas sociales y señala que «lo que aparentemente podría ser un caso de pasividad y docilidad deplorables, desde un punto de vista progresista, puede muy bien ser una forma de agencia social, que debe ser entendida en el contexto de los discursos y las estructuras de subordinación que crean las condiciones de su representación» (p. 188). Por ello, Mahmood distingue el concepto de agencia de los de resistencia y dominación, y sitúa la «agencia como una capacidad para la acción creadora y propiciada por relaciones de subordinación específica» (2008: 133).

Precisamente, este enfoque instala la relación en los procesos de vulnerabilidad (dominación/subordinación) y los procesos de agenciamiento (resistencia) (Willers, 2016). Butler et al. (2016) señalan que tradicionalmente se sitúan ambos procesos como opuestos: la vulnerabilidad es asociada con la victimización e inacción; en cambio, la resistencia se presenta como un factor de transformación social y, por tanto, un espacio donde actúa la agencia. Sin embargo, para estas autoras, la resistencia emerge de la vulnerabilidad, de ahí que consideran necesario desmantelar la mirada dicotómica entre ambos procesos y situar la vulnerabilidad como una dimensión que posibilita la capacidad de agencia y, por tanto, de resistencia (Ferrarese, 2017; Gandarias, 2019). Cabe aclarar que esto no implica que la vulnerabilidad per se conlleve el desarrollo de la agencia, pero facilita descentrar el análisis desde una lógica dicotómica otorgando mayor amplitud y matices a las maneras en que se pueden lograr acciones para transformar aquello que violenta, segrega o vulnera. En esta misma línea, Martínez (2019) propone preguntarse cómo se desarrolla la agencia en situaciones de vulnerabilidad, más que preguntar por la agencia de los vulnerados

Pero ¿qué entendemos por vulnerabilidad? Para Busso (2005), es multidimensional y multicausal, ya que en ella «confluyen simultáneamente la 
exposición a riesgos, la incapacidad de respuesta y adaptación de individuos, hogares o comunidades (...) ante cambios o permanencia de situaciones externas y/o internas que afectan su nivel de bienestar y el ejercicio de sus derechos» (p. 16). Así, la vulnerabilidad está asociada con riesgos y amenazas múltiples que determinan la capacidad de respuesta para anticiparse, sobrevivir o resistir a dinámicas que pueden desencadenar exclusión social (Araujo, 2015). En consideración a esto, la vulnerabilidad social no se circunscribe exclusivamente a un tema de carencia socioeconómica, sino también a la fragilidad institucional y al debilitamiento de las redes sociales, familiares y comunitarias (Araujo, 2015; Lenta y Zaldúa, 2020). Por tanto, situarla como dimensión analítica implica considerar la relación entre el componente individual (emocional y simbólico), el componente social (relacional) y el componente programático (protección de las políticas sociales) (Lenta y Zaldúa, 2020). Esta imbricación permite comprender múltiples factores que actúan en la articulación de dinámicas de vulnerabilidad, pero que, de acuerdo con las aportaciones feministas, serían una fuerza movilizadora, es decir, locus de resistencia contra distintas formas de poder (Gandarias, 2019; Nijensohn y Minici, 2017). Esto último va en línea con aquellos argumentos que sitúan la resiliencia no como una actitud pasiva frente a las desigualdades sociales, sino como una actitud que emerge para desafiar mecanismos subyacentes de exclusión, y así lograr mayores niveles de bienestar personal y colectivo (Hall y Lamont, 2013).

Ahora bien, retomando la relación entre agencia, vulnerabilidad y resistencia, Pollack (2000) cuestiona la manera de tratar los conceptos de víctima y agencia, dicotomía que no permite comprender que las mujeres que enfrentan procesos de victimización han actuado en contextos opresivos. Por su parte, Mahoney (1994) señala que en el caso de las mujeres maltratadas la necesidad de sobrevivir y cuidar a sus hijos e hijas les permite conocer sus propias fuerzas al actuar bajo presión y responder a la opresión con resistencia. En este caso, se ejemplifica la forma en que la agencia y la victimización se definen socialmente de manera dicotómica y excluyente; es decir, a quienes se les concibe como agentes no pueden ser considerados/as como víctimas y viceversa. Sin embargo, siguiendo con este ejemplo, es importante no desconocer las experiencias de las mujeres como víctimas, pero tenerlo como un enfoque exclusivo limita el análisis, pues desconoce los esfuerzos y recursos que movilizan a las mujeres para sobrevivir. Asimismo, un enfoque exclusivo en la agencia que sitúa la acción y deliberación individual como estrategia es insatisfactorio si no se considera el contexto social que conlleva la victimización (Dunn y Powell-Williams, 2007).

La resistencia como dimensión transversal de la agencia (Nadler y Shnabel, 2015) se caracteriza por la acción y oposición para socavar la opresión y subordinación (Hollander y Einwohner, 2004). Así, las resistencias colectivas se pueden situar, por ejemplo, a través de acciones de protesta, y las de carácter individual se pueden observar, por ejemplo, en la actitud de algunas mujeres musulmanas que, en contextos más liberales y donde impera con mayor fuerza la islamofobia, usan el hijab para reafirmar sus identidades religiosas (Mahmood, 2019; Ray Vollhardt et al., 2020). Cabe destacar la complejidad 
del concepto de resistencia, ya que es posible observar que mientras se resiste al poder se pueden estar gestando de forma paralela dinámicas de complicidad, acomodación o conformidad con el mismo poder al que se resiste. Así, Hollander y Einwohner (2004) argumentan la necesidad de no dicotomizar la realidad entre resistencia versus dominación, pues ello construye narrativas que ignoran los múltiples sistemas de opresión donde los individuos pueden estar, de manera simultanea, en relaciones de dominación y resistencia. Se trata, en última instancia, de la estructura que, desde el interaccionismo simbólico, representa las condiciones en las que se desarrolla la acción social.

\section{Mujeres inmigrantes: una aproximación al contexto}

La feminización de las migraciones es considerada una de las características de la actual «era de las migraciones» (Castles y Miller, 1998). Este fenómeno que se caracteriza por emplazar a las mujeres como protagonistas de los movimientos transnacionales (Parella, 2003). En tal sentido, Parella (2005) señala que «una lectura de las migraciones en clave de género permite concluir que la migración femenina ya no puede atribuirse solo al hecho de que las mujeres sigan a sus esposos de forma pasiva, sino que las mujeres emigran a menudo solas y siguen patrones migratorios distintos a los de sus homólogos masculinos» (p. 104). En efecto, la literatura ha puesto de manifiesto la existencia de patrones migratorios diversos (Pérez, 2008): mujeres que emigran como estrategia económica familiar (Oso, 2000; Sassen, 2003); mujeres reagrupadas (Elgorriaga et al., 2012); mujeres que huyen de situaciones de violencia de género (López, 2020), y finalmente las que buscan un cambio de vida (Micolta, 2005).

Esta heterogeneidad influye en las motivaciones para migrar (Elgorriaga et al., 2012), situación que, para Herrera (2002), conlleva no centralizar el análisis en aspectos de carácter económico, pues no explican la totalidad de la migración femenina. Por ello, señala la autora, es necesario incorporar «factores sociales y culturales que no necesariamente responden a una lógica económica racional». «Imaginarios», «redes sociales», «cadenas horizontales y verticales» y «estrategias familiares» son las herramientas conceptuales que se despliegan para ofrecer una interpretación social y cultural antes que económica de la migración» (p. 89-90).

Ahora bien, los estudios sobre migración y genero han permitido analizar temas vinculados a la inserción en el mercado laboral, principalmente en el área de los cuidados (Lebrusán et al., 2019; Parella, 2003), la maternidad transnacional y las cadenas globales de cuidados (Hochschild, 2001), la violencia machista (Moriana, 2018; Arnoso et al., 2012), la sexualización de las mujeres inmigrantes (Wade et al., 2008), las redes migratorias (Pedone, 2002) y las estrategias de producción y reproducción social (Barañamo y Marchetti, 2016).

En España, a partir de la década de los noventa y producto del crecimiento económico, sumado al reparto desigual de las tareas reproductivas dentro del hogar, al aumento de las mujeres autóctonas en el mercado laboral y a la privatización de los servicios de los cuidados (Martínez, 2014), se observa 
un incremento de mujeres inmigrantes, en especial latinoamericanas (Muñoz de Bustillo y Antón, 2010), para suplir estas labores. Se trata de un cambio de trasformación global (Sassen, 2003) que sitúa a las mujeres inmigrantes como mano de obra en «mercados emergentes del trabajo doméstico, los cuidados, los servicios sexuales u otros aspectos de la reproducción social» (Barañano y Marchetti, 2016: 11). Los estudios en torno a la configuración del sector doméstico han permitido corroborar dos cuestiones: por un lado, su invisibilidad social y su normativa diferenciada del régimen general y, por otro, situarlo como una vía de acceso a la regularización administrativa para numerosas mujeres extranjeras (Lebrusán et al., 2019).

A modo general, Oso y Parella (2012) señalan que los estudios relacionados con la incorporación de las personas inmigrantes al mercado laboral han tenido un sesgo de género, pues no han profundizado en los riesgos y vulnerabilidad a los que se enfrentan las mujeres inmigrantes, situación que demanda incorporar la perspectiva de género, incluso en las políticas migratorias (Arnoso et al., 2012).

Otro tema transversal en los estudios migratorios son los procesos y las dinámicas de integración social. Las contribuciones han puesto de manifiesto los obstáculos que encuentra la población migrada para lograr la integración, los cuales se observan en el ámbito «legal (visado, proceso de regulación, ley de extranjería, etc.), económico (nichos laborales, explotación, subempleo, etc.), político (asimilacionista, segregacionista, etc.), antropológico (interculturalidad, conflicto de valores, etc.), psicológico (estrés adaptativo, procesos psicopatológicos, habilidades sociales, etc.)» (Martínez et al., 1999: 222).

En un estudio comparativo entre mujeres inmigrantes magrebíes y latinoamericanas, Elgorriaga et al. (2012) muestran que en ambos colectivos los factores que dificultan la integración son la situación administrativa irregular, la ocupación en un mercado laboral segmentado y etnificado, las dificultades en la homologación de estudios profesionales y las precarias condiciones de vivienda. Para Bruquetas y Moreno (2015), este escenario se complejizó a partir de la crisis económica del 2007, pues se incrementó la vulnerabilidad de la población inmigrante, en especial de las mujeres, al ser uno de los colectivos más afectados por el desempleo, lo cual tuvo un impacto en el estatus legal ya que aumentó la irregularidad sobrevenida. Con relación a esto último, Sánchez (2011) indica que es la propia normativa de la inmigración española la que genera irregularidad administrativa, debido a la interdependencia entre permisos de trabajo y permisos de residencia, que se suma a la burocracia que retrasa los expedientes de las personas inmigrantes.

Por otro lado, entre los factores que facilitan la integración social, destacan las redes de apoyo entre la población migrante y las prácticas asociativas entre mujeres (Elgorriaga et al., 2012). De todos modos, González-Rábago (2014) manifiesta que dicha integración no debe ser entendida de manera unidireccional, sino más bien desde la reciprocidad entre la población autóctona y la población inmigrante, y que es fundamental analizar la implicancia del Estado en dicho proceso. 
Si consideramos los factores mencionados, observamos que las redes actúan en dos direcciones: por un lado, como dinamizador de los circuitos migratorios, ya que transmiten información a las comunidades en el país de origen, y, por otro, como espacio de apoyo y transferencia de información en el país de destino, relacionado principalmente con el acceso a la vivienda y al mercado laboral (Goycoechea y Ramírez, 2002).

Con respecto a las prácticas asociativas entre mujeres, la investigación de Gordano (2017) muestra cómo la participación en espacios asociativos en la ciudad de Barcelona ejerce una influencia entre las experiencias migratorias como mujeres feministas y las experiencias feministas como mujeres migrantes. Esta intersección visibiliza dinámicas de resistencia y agencia que sitúan como eje transversal la participación en redes sociales, políticas y migratorias como mecanismo para sortear las dificultades. Para Sipi (2000), la asociatividad de las mujeres inmigrantes ha desafiado su "supuesta» invisibilidad al desplegar prácticas políticas que buscan reivindicar derechos básicos, pero también exigir una «revisión del racismo y etnocentrismo de los libros de texto escolares para que los niños inmigrantes y de otras minorías étnicas no se avergüencen de sus orígenes; la superación del trato criminalista de los medios de comunicación sobre los inmigrantes» (p. 361).

De esta manera, los estudios sobre migración y género han profundizado en una amplia variedad de temas que muestran una multiplicidad de situaciones y escenarios a los que se enfrentan en el país de destino. Esta investigación contribuye en esta línea, ya que destaca cómo se desarrollan los procesos de agencia en contextos de riesgos y vulnerabilidad.

\section{Aproximaciones metodológicas}

En esta investigación se realizaron historias de vida a 25 mujeres latinoamericanas de Chile, Argentina, Uruguay, Perú, Bolivia, Ecuador, Colombia, México, Costa Rica, República Dominicana y Brasil. Se optó por las latinoamericanas porque son el colectivo de mujeres inmigrantes extracomunitarias con mayor presencia en la ciudad de Barcelona y España (INE, 2017).

La muestra se definió mediante un muestreo intencional centrado en dos criterios de selección: la edad y los años de residencia.

En cuanto a los grupos etarios, se precisaron los tres más representativos en edad laboral: 24 -34 años; 35-44 años y 45 años y más (Parella y Petroff, 2014). En la Comunidad Autónoma de Cataluña, la migración femenina ha aumentado considerablemente, hasta llegar a equipararse a la masculina (INE, 2017). Su presencia en el mercado laboral se concentra en los llamados servicios de proximidad o reproducción social, es decir, actividades remuneradas vinculadas al servicio doméstico y cuidado de personas (Parella, 2003). Cabe puntualizar que las investigaciones en torno a la feminización de las migraciones han constatado que una parte significativa de las mujeres que migran lo hacen como una estrategia económica familiar, lo cual sitúa el trabajo como una dimensión transversal en sus vidas, pues les permite cumplir las deman- 
Tabla 1. Perfil de mujeres entrevistadas

\begin{tabular}{rlrlrl}
\hline N. & Situación administrativa & N. & Nivel de estudios & N. & Ocupación \\
\hline 6 & Situación administrativa irregular & 5 & Estudios universitarios & 12 & Servicio doméstico \\
19 & Situación administrativa regular & 7 & Bachillerato & 6 & Desempleadas \\
9 & Secundarios completos & 2 & Psicólogas & & \\
4 & Secundarios incompletos & 1 & Periodista & & \\
1 & Médica & & & & \\
1 & Abogada & & & \\
2 & Técnica tercer sector & & & & \\
\hline
\end{tabular}

Fuente: Elaboración propia a partir del perfil de la muestra

das económicas en el plano personal y familiar (Barañano y Marchetti, 2016; Martínez, 2014; Parella, 2003; 2005).

Los años de residencia se dividieron en dos intervalos: menos de tres años y más de tres años, pues, según el reglamento de la Ley Orgánica 4/2000 sobre Derechos y Libertades de los Extranjeros en España y su Integración Social, para acceder a la residencia por arraigo social se debe acreditar la permanencia continua en el país por más de tres años, sumada a un informe que demuestre el grado de integración o inserción social del extranjero/a.

Se trabajó con historias de vida ya que, como enfoque biográfico-narrativo, esto permite incorporar la subjetividad de las personas al reconstruir la experiencia vivida y analizar cómo determinados acontecimientos, situaciones o experiencias han influido en sus trayectorias vitales. En efecto, son las narrativas las que dan significado a las experiencias vividas y relatadas (Domínguez y Herrera, 2013). De ahí que profundizáramos en dos momentos. En primer lugar, se abordó la etapa previa a la migración considerando las trayectorias laborales, relaciones familiares, sociales y de pareja, las motivaciones y objetivos migratorios, así como el conocimiento del contexto español y catalán. En segundo lugar, se incidió en las vivencias y experiencias ya en Barcelona: la manera en que se gestionó el desarraigo sociofamiliar, las estrategias de participación social en redes de inmigrantes o en espacios políticos o asociativos, los procesos de regulación administrativa, las dificultades para acceder al mercado laboral, así como también en situaciones de discriminación social y laboral. Durante el proceso de las entrevistas, fueron emergiendo otros temas, como por ejemplo situaciones de acoso sexual en la vía pública o en el ámbito laboral, dificultades con la homologación de los estudios universitarios, problemas de salud mental (depresión y ansiedad), la maternidad transnacional y la violencia de pareja.

Para develar las narrativas, se trabajó con el análisis crítico del discurso feminista (ACDF). Para Dijk (1999), el análisis crítico del discurso (ACD) es «un tipo de investigación analítica sobre el discurso que estudia primeramente el modo en que el abuso de poder social, el dominio y la desigualdad son practicados, reproducidos y ocasionalmente combatidos por los textos y el habla en el contexto social y político» (p. 23). No obstante, las investigadoras feministas 
señalan que el ACD no garantiza una mirada crítica sobre las desiguales relaciones de género. De ahí que el ACDF sea una reapropiación del ACD (Azpiazu, 2015) que busca, por un lado, identificar y analizar los discursos que sostienen un orden social patriarcal y, por otro, clasificar los significados del discurso que vislumbran, sutil o estructuralmente, una disposición a transformar o subvertir relaciones sociales asimétricas que, cruzadas por el género, forjan dinámicas de exclusión (Lazar, 2007). Bacchi (2006) considera necesario analizar los esquemas interpretativos y conceptuales de los discursos que sustentan relaciones de poder. Para ejemplificarlo, cita un estudio de Stapleton y Wilson, que señalan que tanto el género como la nacionalidad se construyen como categorías discursivas que estructuran las identidades del hablante, aunque ello no limita el desarrollo de auto-narrativas como mecanismo para negociar con estas estructuras y, así, no determinar sus identidades discursivas.

De forma paralela, trabajamos con la perspectiva interseccional, pues, para el estudio de las migraciones femeninas, se configura como una herramienta teórico-metodológica que permite analizar las experiencias de desigualdades múltiples (Crenshaw, 1989), al considerar la heterogeneidad de condiciones y posiciones sociales como lo es la clase, la raza/etnia, los circuitos migratorios, los tipos y motivos de la migración, entre otros. Esta conexión facilita y otorga una mayor aproximación al impacto político y social que tiene la subordinación más allá de una lectura centrada en las implicancias del género (Crenshaw, 1989).

Considerando estos dos enfoques, estructuramos el análisis a partir de tres conceptos — vulnerabilidad, estrategias y agencia- que nos permitieron definir dos momentos. En primer lugar, identificar y analizar los factores que han complejizado las trayectorias migratorias en cuanto al acceso al mercado laboral, los procesos de integración social, participación en redes o espacios asociativos, entre otros temas. En segundo lugar, develar cómo estos factores actúan (en parte) como un catalizador en la búsqueda de alternativas que han facilitado, en un primer momento, afianzarse en Barcelona y, posteriormente, redefinir las motivaciones que originaron la migración, aquellas que fueron emergiendo una vez instaladas y los cambios que experimentaron subjetivamente.

\section{Resultados y discusión}

\subsection{El comienzo del devenir migratorio}

La migración es un proceso y un punto de inflexión en las biografías de quienes deciden emigrar, es un momento de ruptura que conlleva cambios y desafíos. En el caso de las mujeres latinoamericanas, considerando el rol que cumplen en las dinámicas sociales y familiares, tanto en origen como en destino (Elgorriaga et al., 2012), estudiar sus trayectorias migratorias implica analizar los cambios personales y subjetivos y de qué manera influyen en el terreno emocional. En este sentido, uno de los temas que emergen del análisis de las historias de vida se relaciona con la soledad y el desarraigo en el periodo de llegada y asentamiento. La falta de pertenencia socioafectiva profundiza sentimientos que, en 
algunas participantes, generó bloqueo existencial, tal y como se expresa en el siguiente relato.

(...) Verme en una ciudad tan desconocida, aunque tú no lo creas, es como que te falta el oxígeno. Estaba sola porque mi marido trabajaba y me recluí en el departamento, porque me decía y ahora qué hago, y me bloqueé. (Lorena, 45 años, procedente de Ecuador, con dos años de residencia en Barcelona)

Si bien los sentimientos que surgen producto de la separación familiar son difíciles de gestionar, los vínculos y el sentido de pertenencia no se difuminan con la distancia, al contrario, en esta primera etapa están muy presentes, aunque su intensidad varía según cada persona. Si las condiciones de llegada y asentamiento son favorables (integración social o laboral), esta ausencia se contrarresta con logros o nuevos aprendizajes; en caso contrario, esto se puede cronificar y dificultar aún más el proceso migratorio.

De manera paralela, en esta etapa comienza una reelaboración de significados y aspiraciones personales que reubica los aprendizajes y experiencias pasadas en un proceso de progresión y regresión; de ambivalencias y certezas que conllevan un esfuerzo por adaptarse a los cambios y por convivir entre dos tiempos y espacios; una lectura dual entre lo definitivo y transitorio, entre la estima y el rechazo, etcétera.

Cuando ya me situé aquí, tuve que comenzar de nuevo; me hice un diagnóstico y me tuve que rearmar como mujer en esta nueva etapa. (Cristina, 29 años, procedente de Chile, con un año de residencia en Barcelona)

El desarraigo (emocional, simbólico y territorial) activa el desarrollo de estrategias que, miradas en clave de agencia, buscan desafiar los cambios, dificultades y limitaciones. Precisamente, los símbolos o referentes subjetivos que lo identifican al país o lugar de procedencia son factores que permiten trazar una reterritorialización que sitúa el cuerpo como territorio que se resignifica para no perder aquello que otorga identidad (McDowell, 2009). Es decir, un ensamblaje simbólico habitado por historias, memorias y conocimientos que confluye en un espacio-tiempo, cuya síntesis define nuevas narrativas que lo reactualizan como un constructo social, con la incorporación simbólica y materialmente del devenir en el que interactúan confluencias múltiples que inciden en la subjetividad. Desde esta lectura, el cuerpo se emplaza como un eje visible de este sujeto-otro que, atravesado por relaciones de poder y por diferentes códigos sociales, culturales y figurativos, absorbe la hibridez que implica la localización de ser un sujeto transfronterizo que busca reinventarse desde la diferencia subvirtiendo los determinantes sociales que lo posicionan en imaginarios estáticos y homogéneos. Así lo indica el siguiente relato.

(...) Me costó comprender que no podía seguir ensoñada con la vida que tuve en mi país, por algo emigré, y debía readecuarme en un nuevo escenario donde renacer, a pesar de lo duro que es vivir como extranjera en una tierra que te 
desconoce. Pero sigo añorando mi tierra. (Estefanía, 52 años, procedente de Ecuador, con 13 años de residencia en Barcelona)

Este extracto muestra cómo la relación con el territorio nacional implica añoranzas de tipo simbólico-afectivo, aunque ello no impide «renacer» mediante una praxis transformadora que sitúa las dificultades como desafíos.

Otro tema que surge de las narrativas es la posición multisituada, en cuanto al contacto permanente entre uno y otro lado de la frontera, y que se ejecuta por medio de acciones y decisiones enmarcadas en un sistema de redes que configuran un espacio relacional no físico. Las interacciones bidireccionales se organizan en el tiempo y el espacio, tanto por el envío de remesas como por el intercambio de ideas y tradiciones que influyen en la reconfiguración de identidades individuales, familiares y sociales. Obviar tales interrelaciones debilita la sostenibilidad de la dimensión práctica y simbólica de lo transnacional. En este sentido, una de las participantes nos otorgó una de las claves para entender esta posición, la cual ha sido recurrente en gran parte de las historias de vida analizadas.

(...) Hoy mi cuerpo está acá y mi mente está allá. Es un constante desdoblamiento y eso me pesa mucho. (Serena, 34 años, procedente de Uruguay, con nueve años de residencia en Barcelona)

La simultaneidad define, desde una mirada diacrónica, un campo social inscrito más allá de los límites geográficos del estado-nación y cuyos nudos se conectan a través del intercambio en un espacio-tiempo construido desde la facilidad que otorgan las redes sociales. La reflexión en torno a la localización es un eje que nos permite profundizar en el carácter subjetivo de la posición/ condición que implica ser mujer inmigrante y los mecanismos que las mujeres desarrollan para «adaptarse» o «integrarse». Pero también aporta elementos para entender cómo se van conformando cartografías que conectan realidades diversas y distantes (Glick Schiller et al., 1992).

La irrupción de relaciones sociales transfronterizas conforma redes que facilitan el intercambio y que acentúan transformaciones subjetivas e identitarias, pues es precisamente la convergencia de un espacio temporal multisituado la que otorga nuevas perspectivas a la hora de sentir las emociones, vivir las relaciones y recrear los vínculos (Barañano y Marchetti, 2016; Glick Schiller et al., 1992). Estas transformaciones permiten situar a las mujeres migrantes como agentes activos frente a una realidad que, en un primer momento, se presenta confusa, pero que luego se vuelve dinámica a causa de las nuevas articulaciones, experiencias y posicionamientos que despliegan una vez asentadas en la ciudad.

Valor y fortaleza se intersectan con la vulnerabilidad que implica vivir en un territorio donde los riesgos son múltiples y latentes. Así lo muestran las siguientes citas.

Yo trabajo en negro cuidando a un abuelo, me ofrecieron hacerme contrato, pero iban a pagar $280 €$ menos y no me convenía (...). Sin contrato no hay derechos, y sin derechos la gente hace lo que quiere contigo. Aprendes a vivir 
con la fragilidad, pero eso te hace más fuerte. Aunque parezca tonto lo que digo, te hace más lúcida y astuta. (Rocío, 44 años, procedente de Bolivia, con siete años de residencia en Barcelona)

(...) Estoy sin papeles y eso es complicado porque no puedes acceder a nada y estás expuesta a muchos riesgos. Que la policía esté haciendo control y me coja es un riesgo, porque me pueden mandar a un CIE o deportar. Te acostumbras a caminar mirando a todos lados. Yo digo: «El riesgo y el miedo son mis amantes, nunca me dejan». (Juana, 38 años, procedente de Colombia, con tres años de residencia en Barcelona)

Justamente, los estudios sobre migración y género han constatado los riesgos a los que se exponen las mujeres, por ejemplo, en el trabajo doméstico. Un diagnóstico realizado por Montero y Rosario (2015), de la Red de Mujeres Latinoamericanas y del Caribe en España, muestra cómo la precariedad, la irregularidad y la desprotección social son una fuente de abusos que las expone a situaciones de fragilidad social. Parella (2003) añade que, si bien los servicios de proximidad en Catalunya son una oportunidad de inserción laboral, se caracterizan "por los bajos ingresos, malas condiciones de trabajo, el escaso grado de organización colectiva, la precariedad laboral, los altos índices de economía sumergida y las limitadas posibilidades de promoción laboral» (p. 110). La irregularidad administrativa es otra fuente de vulnerabilidad, pues quienes no ostentan la condición de ciudadanos/as pierden el reconocimiento como sujeto político y, por tanto, sus derechos. Esta situación institucionaliza la exclusión de la población inmigrante al estar condicionada por el estatus migratorio (Merino, 2017).

\subsection{El contexto migratorio: violencias y desigualdades}

Los primeros años de residencia revisten un proceso de adaptación a dinámicas sociales, políticas, culturales y económicas muy diferentes a las vividas en sus países de origen. Sin embargo, existen factores que obstaculizan la integración o adaptación. Un ejemplo de ello lo observamos en el ámbito laboral, donde el descenso en la escala social se debe principalmente a las dificultades en el proceso de homologación de sus estudios profesionales, y además al hecho de que la oferta laboral se concentra especialmente en el trabajo doméstico y de cuidados, el cual es desvalorado social y económicamente. Sumado a ello, las mujeres latinoamericanas deben enfrentar el enquistamiento de una imagen estereotipada de los roles de género que promueve su integración casi exclusivamente a tareas reproductivas (Parella, 2003). De esta forma, son múltiples los factores que pueden ralentizar la inclusión social o, peor aún, agudizar dinámicas de exclusión.

Explorar en este complejo escenario nos advierte de situaciones que definen un entramado social que incrementa situaciones de vulnerabilidad. En este sentido, una de las características de la estructura social es la tendencia a establecer procesos de diferenciación y categorización hacia la población inmi- 
grante estableciendo fronteras simbólicas y jurídicas entre esta y la población autóctona. Para situar dicho proceso, es oportuno visualizar de qué manera las leyes o prácticas sociales fundan grados de segregación, y hasta qué punto ello conlleva el desarrollo de dinámicas que promueven el rechazo o discriminación (González-Rábago, 2014). En el caso de esta investigación, observamos un andamiaje que se expresa en tres espacios-tiempos: el económico, que gira en torno al abuso, explotación y precarización laboral que se concentra en un mercado segmentado y etnificado; el político/institucional, que se expresa mediante la irregularidad administrativa y limitación de derechos, y el simbólico, que se estructura a través de los estereotipos, prejuicios, homogenización, negación epistémica y violencia. Tales áreas se formulan y expresan de maneras diversas y no siempre mantienen la misma intensidad y continuidad; se dilatan y contraen, pero ello no significa que se amortigüen su finalidad y sentido procesual. Es más, son estas variaciones las que otorgan - desde una perspectiva multidimensional — complejidad a las dinámicas de exclusión social. Por tanto, la segregación habitacional o social y la limitación de derechos civiles y políticos producto de la situación administrativa irregular son parte de las dificultades o situaciones que acentúan la vulnerabilidad. Así lo ejemplifican dos participantes.

(...) Una cosa que me descolocó fue ser consciente de que eras la "otra». Yo pensaba que al venir aquí siendo blanca, argentina, con apellido italiano, además de ser médica, estas cosas no se iban a dar y, sin embargo, sí que se dieron. (Estrella, 32 años, procedente de Argentina, con cinco años de residencia en Barcelona)

(...) Partiendo de que no tengo papeles y no puedo trabajar, ya me siento discriminada, además soy negra y pobre, y eso no ayuda mucho. (Berta, 33 años, procedente de la República Dominicana, con dos años y medio de residencia en Barcelona)

Relatos que ilustran hasta qué punto los procesos de exclusión pueden ser leídos desde una mirada interseccional al imbricarse la condición de migrante, la raza (color de piel), el género y la clase social, y que, más allá de los discursos institucionales que buscan la integración, existen factores que fomentan prácticas o mecanismos que provocan desigualdades que se superponen y entrecruzan de forma simultánea y recíproca (Smith, 1983). De este modo, la migración se sitúa como un campo reflexivo donde, si bien existe exclusión, hay una disposición a transformarla amortiguando las incertidumbres y buscando respuestas o soluciones prácticas.

Como inmigrante sin papeles y mujer negra, tuve que trabajar como una esclava, 12 horas al día por $30 €$. El trato era de lo peor, y si me quejaba, irónicamente, la dueña de la casa me decía: «Anda a denunciarme, a ver qué te pasa». Busqué otro trabajo, pero antes de irme tuve el placer de decirle en su cara lo racista que era. (Ester, 40 años, procedente de Bolivia, con ocho años de residencia en Barcelona) 
La mirada crítica y reflexiva de la participante sitúa la negritud como una categoría que inferioriza, añadida a la dificultad que significa no contar con permiso de residencia, factores que limitan el acceso a determinados derechos y oportunidades (Oso y Parella, 2012). El racismo opera como forma de exclusión social, un desprecio por aquello considerado inferior, donde el miedo al rechazo y la inseguridad permean la subjetividad de quienes lo viven (Fanon, 2010). Las mujeres afirman que este tipo de situaciones afectan su autoestima y que, incluso, las lleva a cuestionarse el hecho migratorio, pero ello no limita la búsqueda de alternativas o estrategias para sortear situaciones de violencia. Así, frente a las experiencias de exclusión, se observa una disposición por buscar espacios de inclusión que, de manera sincrónica, aportan posibilidades de acceso en distintas áreas. En este sentido, las acciones emprendidas individualmente corroboran una actitud por sortear situaciones que las hacen más vulnerables.

(...) Después de muchas situaciones de violencia en los trabajos que he tenido aquí en Barcelona, me acerqué a una asociación de mujeres inmigrantes que asesoraba a las trabajadoras. Tengo la suerte de tener papeles y puedo luchar por mis derechos. (Serena, 34 años, procedente de Uruguay, con nueve años de residencia en Barcelona)

(...) De lo malo tienes que sacar lo bueno, de lo contrario, te puedes hundir en la depre. Ser migrante no es nada fácil, pero aprendes o valoras tu propia fortaleza, es cuando dices: «iNunca pensé que podría hacerlo, y lo hice!». Y esa ha sido una tremenda ganancia. (Rosa, 38 años, procedente de Perú, con ocho años de residencia en Barcelona)

Esto último entra en sintonía con Butler (2014) cuando subraya que la vulnerabilidad activa procesos de transformación, pues actúa como fuerza que, consciente o inconsciente, busca subvertir el horizonte «normativo. Es más, Butler (2015) señala que durante mucho tiempo se consideró que las mujeres eran especialmente vulnerables, afirmación que justifica disposiciones paternalistas sobre las mujeres: "Esta visión es muy diferente a la que afirma, por ejemplo, que las mujeres son a la vez vulnerables y capaces de resistir, y que la vulnerabilidad y la resistencia pueden y deben, e incluso, ocurrir al mismo tiempo» (p. 140). En esta misma línea, la resiliencia, que muchas veces es entendida como pasividad frente a la violencia y la desigualdad, es una actitud que pretende transformar, a través de prácticas individuales o colectivas, aquello que oprime (Hall y Lamont, 2013).

Por otra parte, y como complemento de lo anterior, se observa el rol que ejercen las redes migratorias como pilar de información y soporte social y, en ocasiones, emocional. En el país de origen, las expectativas de las mujeres y su decisión de emigrar son reforzadas por redes que, para el caso de esta investigación, están conformadas por familiares y amigos/as. Una vez instaladas en la ciudad, las redes otorgan la posibilidad de establecer vínculos sociales que amortiguan los conflictos emocionales producto de la separación y el distanciamiento familiar, pero también brindan información sobre las posibilidades 
laborales, los requisitos para regularizar la situación administrativa o el circuito de entidades del tercer sector que ofertan cursos de formación sociolaboral. Un ejemplo de cómo las redes se conforman como espacio de soporte y apoyo lo ejemplifica el siguiente relato.

(...) Aquí tenía a mi familia y a mis amigos y no me sentí sola. Aquí tenía a toda mi gente; en ese periodo no había soledad (...), nadie se podía sentir solo; te llevaban a los bares, te llevaban por allí, te enseñaban, te daban dinero para que te compres tu tarjeta. Normalmente era tu gente, tus tíos, hermanos, etcétera; siempre había mucho apoyo. (María, 51 años, procedente de República Dominicana, con 15 años de residencia)

Para autores como Subirats (2010), la inclusión social se percibe mientras exista participación activa en tres áreas importantes: primero, en la economía por medio de la producción de valor social (dentro o fuera del mercado); segundo, en la política a través del ejercicio de la ciudadanía; tercero, en la esfera social y familiar mediante la pertenencia comunitaria. En efecto, Elgorriaga et al. (2012) indican que las redes facilitan los procesos de integración e inciden en el desarrollo de una ciudadanía activa que trasciende los límites de la nacionalidad, pues en las comunidades de referencia es donde se desarrollan prácticas de organización. Ahora bien, para que deje de ser un oxímoron, Suárez (2006) subraya que es necesario cambiar el modelo de soberanía nacional, que «restringe la incorporación de los extranjeros como ciudadanos de pleno derecho en sociedades democráticas» (p. 29). Precisamente, la autora se pregunta cuál es el reto que plantean los y las inmigrantes a la ciudadanía, y la respuesta viene dada por el reconocimiento del sujeto político que actúa y crea pertenencia en sus comunidades de referencia.

Así, la manera en que las mujeres participantes en esta investigación ejercen la ciudadanía en sus comunidades de referencia es mediante el desarrollo de estrategias que les permiten lo siguiente: potenciar la participación en el tejido asociativo, ampliar redes sociales y establecer vínculos con otras mujeres migrantes; fortalecer el empoderamiento individual y colectivo, y reivindicar derechos como dispositivo para enfrentar la estratificación de oportunidades. Esto se traduce en una capacidad de generar pertenencia y resistencia a un modelo que actúa como mecanismo de exclusión. Es el propio contexto migratorio el que las empuja a construir estas alternativas. En algunos casos, comienza como un acto de supervivencia y, en otros, es un continuo político de lucha por la equidad y justicia social.

Entre las experiencias de participación de las mujeres migrantes, uno de los factores que las limitan es la jornada laboral, por su intensidad y extensión horaria (Monteros-Obelar, 2017). No obstante, y a pesar de ello, las desarrollan por canales «sui generis, como la conformación de redes de apoyo entre quienes comparten un mismo origen nacional o regional, una adscripción religiosa o simplemente la unión que produce experimentar situaciones similares» (p. 154). Un ejemplo de ello lo podemos ver en la siguiente cita. 
(...) Te relacionas primero con tus paisanas, buscas gente de tu país para sentirte menos sola, pero luego, por lo pronto en mi caso, necesité otro tipo de redes y espacios porque fui cambiando. Hoy participo en mi barrio (Nou Barris), hay mucha población inmigrante, pero participan muy pocos (...). Mi filosofía es: "Aquí vivo y aquí me organizo». Me gustaría hacerlo con más frecuencia, pero no puedo porque llego de noche a casa. Hago lo que puedo porque lo que no me falta es actitud y fortaleza. (Rosa, 38 años, procedente de Perú, con ocho años de residencia en Barcelona)

A la hora de sistematizar estas experiencias, hemos detectado tres espacios asociativos de participación. En primer lugar, los colectivos conformados por migrantes de la misma nacionalidad, por ejemplo, las comunidades ecuatorianas, bolivianas o peruanas conforman un sustento para sus connacionales. Estas tres comunidades, con una alta presencia en la ciudad de Barcelona, destacan por su nivel de asociatividad, ya que organizan actividades para celebrar festividades nacionales, acciones de apoyo económico e incidencia en espacios institucionales orientadas a lograr aumentar el acceso a recursos (subvenciones) o mediar en situaciones de riesgo de exclusión social (problemas relacionados con la vivienda, por ejemplo). En segundo lugar, las asociaciones de mujeres inmigrantes, un espacio que, como parte del tejido del tercer sector, tiene un papel fundamental en la defensa de sus derechos, además de ofrecer apoyo psicológico y laboral y de ofertar cursos de formación que facilitan el acceso al mercado laboral. Un ejemplo de ello es la asociación Mujeres Pa'lante, que, con sede en la ciudad de Barcelona y en Hospitalet, merece un reconocimiento por parte de las mujeres en este sentido. Y, en tercer lugar, las asociaciones y colectivos feministas, donde la participación tiene una connotación más reivindicativa, frente a la lucha contra los centros de internamiento de extranjeros/as y la abolición de la ley de extranjería, o de crítica al feminismo blanco por no reconocer sus demandas y luchas.

(...) Al comienzo fue complicado conectar con espacios feministas, porque en las discusiones nunca hablaban de las mujeres presas o de las temporeras de Huelva, no sé, yo me preguntaba de qué mujeres hablan o en qué mujeres piensan, porque claramente las migrantes no estamos ahí. (Sofía, 32 años, procedente de Chile, con cinco años de residencia en Barcelona)

Las dinámicas sociales a las que se han enfrentado las participantes de esta investigación han sido diversas y complejas. Sus experiencias transitan de espacios inclusivos a realidades excluyentes y viceversa, pero a pesar de ello sus prácticas imprimen un sello de esfuerzo que ha generado transformaciones en la forma de ver, entender y actuar en la realidad que habitan. Esto es leído como un proceso de agenciamiento, ya que otorga un valor añadido — por los obstáculos presentes - a la capacidad y la disposición para actuar, de modo que recupera la decisión y el poder efectivo en función de los propósitos individuales y colectivos (Casado, 2003). 


\subsection{Hacia una construcción tipológica de agencia}

Analizar las experiencias del proceso migratorio en mujeres latinoamericanas nos ha permitido aproximarnos a las principales dificultades que enfrentan y a los cambios subjetivos que experimentan. Con relación a esto último, el desarraigo social, familiar, nacional e identitario actúa como un componente que incrementa e intensifica la complejidad de las trayectorias migratorias. A pesar de ello, tal y como señalan Domínguez y Contreras (2017), es necesario cuestionar «la construcción homogénea que se ha hecho de la mujer migrante, un arquetipo que se define desde la fragilidad, vulnerabilidad e indefensión y que funda estereotipos y estigmatizaciones que invisibilizan su heterogeneidad y niegan su capacidad agencial» (p. 80). Aquí se instala la riqueza analítica de la agencia, pues favorece el análisis sobre el rol activo y protagónico de las mujeres en la transformación del patrón de movilidad, en la resignificación de sus identidades, en los cambios personales, familiares y sociales que implica el vivir transnacional, la manera en que comienzan a consolidar acciones tendientes a enfrentar las dificultades y confrontar imaginarios o estereotipos construidos alrededor de la figura del sujeto femenino migrante (Casado, 2003; Mahmood, 2008).

Ahora bien, como resultado de la saturación de los discursos, a continuación presentamos una tipología de tres formas de agenciamiento que, a partir de la intersección de factores tales como la edad, los años de residencia, el nivel formativo, el país de procedencia y el color de piel (raza), logran generar procesos de transformación personal. Cabe destacar que esta tipología no está construida desde una mirada jerárquica, sino que representa la actitud/disposición que van definiendo las mujeres en función del momento, el contexto o las situaciones que les toca resolver.

\section{a) Aprendizaje y desmontaje}

Se cruza con los años de residencia y el conocimiento de la realidad social. Algunas mujeres experimentaron durante los primeros meses e incluso años un bloqueo hacia la ciudad y hacia quienes la habitan. Ello las llevó, como primera acción, a desarrollar un proceso de aprendizaje de las nuevas pautas sociales y culturales para adquirir herramientas que las ayudaran a desenvolverse en el nuevo contexto social. Para quienes no contaban con redes sociales o familiares al llegar, el proceso fue más complejo, pero la necesidad de supervivencia las arrojó a buscar este aprendizaje. Por otro lado, el miedo al rechazo al comprender lo que implica y significa socialmente ser migrante, sumado a la falta de pertenencias identitarias, fortaleció una actitud distante hacia la población autóctona. Sin embargo, y transcurrido un tiempo, las experiencias personales permitieron desmontar ideas preconcebidas sobre la población catalana y su relación con la migración. De primera impresión, esto puede parecer simple, pero es un cambio subjetivo que permitió el desarrollo de posicionamiento autónomo que implicó ratificar, rechazar o matizar dichas presunciones. 
El proceso de aprendizaje y desmontaje fue simultáneo, pero la capacidad de actuación fue dispar entre las participantes debido a las motivaciones y herramientas personales, aunque observamos acciones transversales que apuntan a la acumulación de experiencias y vínculos que propician reflexiones en torno a su posición en la nueva realidad habitada. Así, por ejemplo, una de las cuestiones que más destacan es aprender el catalán. Si bien el castellano se usa indistintamente, las participantes consideraron que aprender el idioma era una herramienta para conocer el medio social y su inserción laboral. A ello se añade la participación en grupos de conversación con catalanohablantes que les aportó seguridad y conocimiento situado de las opiniones (de una parte) de la sociedad catalana sobre la población migrante.

Para situar este tipo de agencia, exponemos una cita que lo clarifica.

(...) Sentí mucha inseguridad cuando llegué, tenía miedo a que me identificaran como inmigrante, una sensación que no me permitía vivir con soltura y libertad. Por eso, a pesar del miedo, me apunté a estudiar catalán, quería aprender el idioma, pero, sobre todo, quería demostrarme que podía y ese fue el primer paso para romper mis miedos. Yo soy profesora y tengo capacidades. Ponerme ese desafío me ayudo a reconocer que esta es mi nueva vida. Han pasado los años y cuando miro hacia atrás veo lo valiente que fui y sigo siendo porque ese fue el primer paso de mucho. (Andrea, 48 años, procedente de Ecuador, con 12 años de residencia en Barcelona)

\section{b) Dis/posición a revertir}

Se relaciona con los prejuicios y estereotipos vinculados con el país de procedencia y la racialización. La imagen de América Latina como lugar donde se exacerba la pobreza y exclusión justifica la imagen de mujeres que por necesidad pueden tolerar abusos. Si a estas figuraciones se añade la incidencia que tiene el color de piel como resabio colonial (Fanon, 2010), que inferioriza y subordina, la situación se complejiza. En este contexto, algunas entrevistadas manifestaron que, en ciertos espacios sociales, se han sentido excluidas bajo discursos que niegan sus habilidades para entablar una conversación en igualdad de condiciones. Esta situación también se refleja en las precarias y abusivas condiciones laborales. Quienes trabajan en el servicio doméstico señalan que con frecuencia los empleadores/as destacan que las ofertas y condiciones laborales a las que pueden acceder son muy acordes con su situación de mujeres pobres, «ya que si sus países exportaban este tipo de mano de obra no podían pretender tener mejores puestos de trabajo», señala una de las participantes.

Estas y otras experiencias activaron un proceso de agenciamiento mediante una disposición a revertir, un cambio que buscó subvertir el carácter situado de las diferencias por ser mujeres, migrantes, pobres y racializadas. Esta transformación va acompañada de un proceso reflexivo que profundiza en las fortalezas y debilidades personales. Posicionarse frente a dichas situaciones es un cambio subjetivo que da valor a la existencia y a la propia voz. Así, por ejemplo, cuando las participantes dicen "no acepto tus abusos», "tengo argumentos para participar en esta discusión» o "por qué no me escuchan cuando 
hablo", grafican la actitud a recuperar el lugar en la enunciación que le ha sido negada, a valorar la condición de inmigrante, pero sobre todo a entender que, si bien la migración es una válvula de escape a la precariedad económica de sus países, ello no implica asumir desde la conformidad los abusos que han tenido que enfrentar y desafiar.

Para situar este tipo de agencia, exponemos una cita que lo clarifica.

(...) Estar sola y con trabajos tan precarios me hizo caer en una depresión. Un día una vecina andaluza me dio el número de Mujeres Pa’lante y me dijo: «Llama, aunque sea para saber dónde están y hablar con tus paisanas». Sin dudar lo hice, llegué a Hospitalet y me desahogué, ellas me dieron cita con la psicóloga y luego me orientaron para saber cómo sacar la residencia por arraigo social. También hice el curso de catalán con ellas y conocí a buenas amigas. Todo fue como una rueda de cosas positivas, no sé cómo explicarte, pero esa vecina me dio la clave. Desperté y yo comencé a cambiar mi futuro. (Sofía, 32 años, procedente de Chile, con cinco años de residencia en Barcelona)

\section{c) Apropiación y pertenencia}

El tercer tipo de agenciamiento se desarrolla a través de una apropiación activa mediante una disposición a conocer y asociarse en distintos espacios y entidades de la ciudad. Esto surge al entender el lugar que socialmente es asignado a la subalterna; se reconocen sus cuerpos, pero no sus voces, son otras las que representan sus demandas. La subalterna, al estar situada en el campo de las representaciones discursivas, no puede proponer soluciones o respuestas a sus problemas, sino que recibe ayudas y subvenciones desde una lógica paternalista. Esta lectura, con algunos matices, fortaleció una disposición a generar alianzas. Algunas comenzaron a colaborar en colectivos feministas, en asociaciones de personas inmigrantes o entidades del tercer sector como voluntarias, una característica que se replica en los discursos analizados. De igual modo, y desde una mirada pragmática, la manera de afrontar las dificultades fue a través del despliegue de sus habilidades, recursos formativos y el propio capital cultural, herramientas que facilitaron el desarrollo social.

Para situar este tipo de agencia, exponemos una cita que lo clarifica.

Yo venía de una participación en el movimiento feminista en Uruguay, pero la migración me llevó a entender que la militancia feminista es distinta porque ser migrantes es difícil. Lo primero que hice fue ir a Ca la Dona, pero no me sentí cómoda. Luego, conocí a un colectivo feminista de mujeres latinoamericano y comencé a participar en charlas y actividades que ellas organizaban y que eran de formación política. Una militancia que me hacía tener pertenencia y me hizo ampliar mi red y sentirme más segura en la ciudad. (Serena, 34 años, procedente de Uruguay, con nueve años de residencia en Barcelona)

Esta tipología se despliega de manera circular y de ella emerge, de manera subyacente, la performatividad como proceso subjetivo que implica transformar o subvertir - mediante la acción y enunciación — situaciones de violencias o vul- 
Figura 1. Tipos de agenciamiento

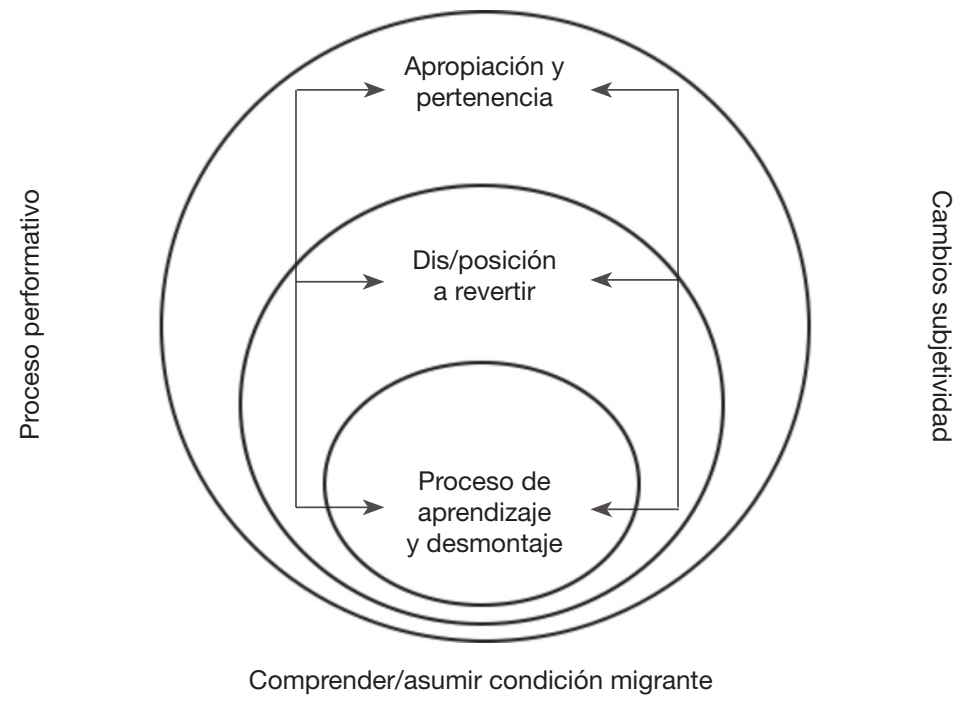

Fuente: elaboración propia.

nerabilidad. La performatividad, por tanto, comporta una dimensión vinculada a experiencias situadas y corporizadas que deviene en una disposición subjetiva y práctica. Desde esta perspectiva, habitar el campo social desplaza fronteras simbólicas que marginan y diferencian y que producen determinado modo de ser. El cuerpo es portador de subjetividad política, pues cobija prácticas y experiencias de dominación, pero también de liberación (Figueroa-Grenett, 2018).

A continuación, se presentan las conclusiones, en las que reflexionamos sobre la agencia y analizamos hasta qué punto ello puede simbolizar un cambio epistemológico para aproximarnos al estudio de las migraciones femeninas.

\section{Conclusiones}

A lo largo del artículo, se resaltan momentos o etapas del proceso migratorio que ofrecen una perspectiva longitudinal que permite observar de qué manera se amalgaman situaciones y emociones que van generando cambios personales, sociales y familiares. De ahí la importancia de las historias de vida como método para acceder a la información, pues permitió que las participantes desarrollaran una reflexión de proceso, en la medida en que se iban articulando los temas que guiaban las investigadoras. Por otro lado, facilitó el análisis y la posterior saturación del discurso en temas convergentes, principalmente los vividos en la ciudad de Barcelona.

En este sentido, al desarrollar una lectura sobre la agencia en contextos migratorios, observamos una articulación de experiencias y proyectos que sitúa 
a las mujeres como sujetas autónomas que intervienen y cuestionan desde los márgenes, protagonistas y promotoras de dinámicas que transforman desde estrategias que confieren una connotación política al sujeto.

Justamente, han sido estas experiencias las que han favorecido el desarrollo de un proceso performativo que proyecta desafíos en un escenario donde la multidimensionalidad de factores sociales, culturales, raciales y de clase configura una red articulada que define relaciones jerárquicas y violentas que refuerzan practicas históricas de dominación y exclusión. Al comprender la construcción ideológica del género y la negación epistémica de la subalterna (Mahmood, 2019; Mohanty, 2008), la agencia permite abordar los dispositivos que las niegan, excluyen o ignoran como sujetos políticos e históricos. Por tanto, su alcance epistemológico se imprime como un código transformador que cuestiona los estereotipos e imaginarios esencialistas, que instala las resistencias como eje que moviliza a las mujeres a la hora de enfrentar la vulnerabilidad.

Por último, respecto a la tipología de agencia, es importante señalar la limitación que proyecta. Si bien esta surge de las historias de vida de las participantes donde, al sistematizar la información, se logró visualizar prácticas y actitudes que buscaban enfrentar y superar situaciones de violencias en contextos de vulnerabilidad, ello puede significar una limitación a la hora de proyectarla en otros contextos o colectivos de personas migrantes, pues las experiencias pueden ser similares, pero las respuestas pueden ser distintas. Por ello, esta tipología surge de las trayectorias del colectivo latinoamericano, considerando que fue una pequeña muestra que tampoco llegó a ser representativa de un colectivo heterogéneo, tanto por la conformación histórica como por la diversidad de motivos y objetivos migratorios. Sin embargo, creemos que es un avance a las aportaciones que se vienen realizando en torno al reconocimiento del sujeto migrante más allá de la construcción pasiva o victimista que lo despoja de su agencia.

\section{Referencias bibliográficas}

Araujo, Rafael (2015). «Vulnerabilidad y riesgo en salud: ¿dos conceptos concomitantes?». Novedades en Población. Cedem, XI (210), 89-96.

Arnoso, Maite; Arnoso, Ainara; Mazkiaran, Mikel e Irazu, Ainhoa (2012). «Mujer inmigrante y violencia de género: factores de vulnerabilidad y protección social». Migraciones, 32, 169-200.

Azpiazu, Jokin (2015). "Análisis crítico del discurso con perspectiva feminista». En: Mendia, Irantzu; LuxÁn, Marta; Legarreta, Matxalen; GuZMán, Gloria; Zirion, Iker y AzPiazu Carballo, Jokin (eds.). Otras formas de reconocer. Reflexiones, herramientas y aplicaciones desde la investigación feminista. San Sebastián: Hegoa-SimRef.

BACCHI, Carol (2006). «Discourse, discourse everywhere: subject "agency" in feminist discourse methodology". Nora-Nordic Journal of Feminist and Gender Research, 13 (3), 198-209.

Barañano, Margarita y Marchetti, Sabrina (2016). «Perspectivas sobre género, migraciones transnacionales y trabajo: rearticulaciones del trabajo de reproducción social y de cuidados en la Europa del Sur». Investigaciones Feministas, 27 (1), 9-34. <https://doi.org/10.5209/rev_INFE.2016.v7.n1.53094> 
Bruquetas, María y Moreno, Francisco (2015). «Precarización y vulnerabilidad de la población inmigrante en la España en crisis. El papel del Estado de bienestar». Panorama Social, 22, 139-151.

Busso, Gustavo (2005). Pobreza, exclusión y vulnerabilidad social: usos, limitaciones y potencialidades para el diseño de políticas de desarrollo y de población. Cepal/Celade.

ButLer, Judith (2014). "Rethinking vulnerability and resistance». <http://bibacc. org/wp-content/uploads/2016/07/Rethinking-Vulnerability-and-ResistanceJudith-Butler.pdf>.

- (2015). Notes toward a performative theory of assembly. Cambridge: Harvard University Press.

Butler, Judith; Gambetti, Zeynep y Sabsay, Leticia (2016). Vulnerability in resistance. Durham: Duke University Press.

Cano, Mónica (2017). «Políticas feministas no identitarias. La agencia desde la deconstrucción y la imperceptibilidad». Revista Internacional de Filosofía, 19, 45-55.

CASAdo, Elena (2003). «La emergencia del género y su resignificación en tiempos de los "post"». Foro Interno, 3, 41-65.

Castles, Stephen y Miller, Mark (1998). The age of migration international population movements on the modern world ( $2^{\mathrm{a}}$ edición). Nueva York: The Guilford Press.

Crenshaw, Kimberlé (1989). "Demarginalizing the intersection of race and sex: a black feminist critique of antidiscrimination doctrine, feminist theory and antiracist politics». University of Chicago Legal Forum, 1, 139-167.

Dıjк, Teun van (1999). «El análisis crítico del discurso». Anthropos, 186 (24), 23-36.

Domínguez, Màrius y Contreras, Paola (2017). «Agencia femenina en los procesos migratorios internacionales: una aproximación epistemológica». Empiria. Revista de Metodología y Ciencias Sociales, 37, 75-99. <https://doi.org/10.5944/empiria.37.2017.18977>

Domínguez, Elsy y Herrera, José (2013). «La investigación narrativa en psicología: definición y funciones». Psicología desde el Caribe, 30 (3), 620-641.

Dunn, Jennifer y Powell-Williams, Melissa (2007). "“Everybody Makes Choices”». Victim Advocates and the Social Construction of Battered Women's Victimization and Agency». Violence Against Women, 13 (10), 977-1.001. <https://doi.org/10.1177/1077801207305932>

Elgorriaga, Edurme; Arnoso, Ainara; Martínez, Cristina y Otero, Nekane (2012). «Proceso migratorio y ajuste psicológico de las mujeres latinoamericanas y magrebíes: un análisis desde la perspectiva de género». Migraciones, 31, 125-154.

Fanon, Frantz (1952). Piel negra, máscaras blancas. Madrid: Akal, 2010.

Ferrarese, Estelle (2017). The Politics of Vulnerability. Londres: Routledge.

Figueroa-Grenett, Claudio (2018). «La acción política de niños, niñas y jóvenes en Chile: cuerpos, performatividad y producción de subjetividad». Revista Latinoamericana de Ciencias Sociales, Niñez y Juventud, 16 (1), 199-212. <https://doi.org/10.11600/1692715x.16111>

GANDARIAS, Itziar (2019). "Resistir desde la vulnerabilidad: narrativas de mujeres subsaharianas sobre su tránsito hacia Europa». Papeles del CEIC, 1-18. <https://doi.org/10.1387/pceic.19532>

Glick Schiller, Nina; Basch, Linda y Blanc-Szanton, Cristina (1992). "Transnationalism: A new analytic framework understanding migration». Annals of the New York of Sciences, 645, 1-24. <https://doi.org/10.1111/j.1749-6632.1992.tb33484.x> 
GonZÁlez-RÁBAno, Yolanda (2014). «Los procesos de integración de personas inmigrantes: límites y nuevas aportaciones para un estudio más integral». Athenea Digital, 14 (1), 195-220.

<https://doi.org/10.5565/rev/athenead/v14n1.1067>

Gordano, Cecilia (2017). «Feminist birds of passage: feminist and migrant becomings of Latin American Women in Spain». European Journal of Women's Studies, $1-16$. <https://doi.org/10.1177/1350506817741324>

Goycoechea, Alba y Ramírez, Franklin (2002). «Imaginarios, familia y redes sociales en la migración ecuatoriana a España (1997-2000)». Íconos, 14, 32-45.

Güell, Berta; Arrasare, Marina y Solé, Ariadna (2020). «Visibilizando estrategias de agencia y empoderamiento económico: el caso de las mujeres de origen pakistaní en Barcelona». Migraciones, 48, 51-78. <https://doi.org/10.14422/mig.i48y2020.003>

Hall, Peter y Lamont, Michele (2013). Social Resilience in the Neoliberal Era. Cambridge: Cambridge University Press.

Herrera, Gioconda (2002). «La migración vista desde el lugar de origen». ÍconosRevista de Ciencias Sociales, 15, 86-94.

Hochschild, Arlie (2001). «Las cadenas mundiales de afecto y asistencia y la plusvalía emocional». En: Giddens, Anthony y Hutton, Will (eds.). En el límite: la vida en el capitalismo global. Barcelona: Kriterios Tusquets.

Hollander, Joselyn y Einwohner, Rachel (2004). «Conceptualizing resistance». Sociological Forum, 19 (4), 533.554. <https://doi.org/10.1007/s11206-004-0694-5>

Instituto Nacional de Estadística (INE, España) (2017). «Cifras de Población al 1 de enero de 2017». <https://www.ine.es/prensa/cp_2017_p.pdf>.

Lazar, Michelle (2007). «Feminist Critical Discourse Analysis: Articulating a Feminist Discourse Praxis». Critical Discourse Studies, 4 (2), 141-164. <https://doi.org/10.1080/17405900701464816>

Lebrusán, Irene; Cáceres, Pierina y Brey, Elisa (2019). «El servicio doméstico como vía de acceso a la regularización administrativa en España». Anuario CIDOB de la Inmigración 2019, 246-272. <https://doi.org/10.24241/AnuarioCIDOBInmi.2019.246>

Lenta, María y ZaLdúa, Graciela (2020). «Vulnerabilidad y exigibilidad de derechos: la perspectiva de niños, niñas y adolescentes». PSYKHE, 29 (1), 1-13. <https://doi.org/10.7764/psykhe.29.1.1225>

Ley Orgánica 4/2000, de 11 de enero, sobre Derechos y Libertades de los Extranjeros en España y su Integración Social. <https://www.boe.es/buscar/act.php?id=BOE-A-2000-544>.

López, Roberto (2020). «Emigración forzada en familias por la violencia en el sur de Sinaloa: experiencias trágicas y complejas». Secuencias, 108, 1-27. $<$ https://doi.org/10.18234/secuencia.v0i108.1727>

Maнmood, Saba (2008). «Teoría feminista y el agente dócil: algunas reflexiones sobre el renacimiento islámico en Egipto». En: SuÁrez, Liliana y Hernández, Rosalva (eds.). Descolonizando el feminismo. Teorias y prácticas desde los márgenes. Madrid: Cátedra.

- (2019). «Teoría feminista y el agente social dócil: algunas reflexiones sobre el renacimiento islámico en Egipto». Papeles del CEIC, 1-31. <https://doi.org/10.1387/pceic.20282> 
Mahoney, Martha (1994). «Victimization or Oppression? Women's Lives, Violence and Agency». En: Fineman, Martha y Mykitiuk, Roxanne (eds.). The public nature of private violence: the discovery of domestic abuse. Londres: Routledge.

Martínez, María (2019). «Una (breve y no sistemática) aproximación a la noción de agencia desde la vulnerabilidad».. Papeles del CEIC, 1-9. <https:// doi.org/10.1387/pceic.20616>

Martínez, Raquel (2014). “¡El trabajo doméstico cuenta! Características y transformaciones del servicio doméstico en España». Migraciones, 36, 275-305. <https://doi.org/10.14422/mig.i36.y2014.002>

Martínez, Manuel; García, Manuel y Jariego, Isidro (1999). «El papel de los recursos sociales naturales en el proceso migratorio». Intervención Psicosocial, 8 (2), 221-232.

McDowell, Linda (2009). Working bodies: interactive service employment and workplace identities. Oxford: Wiley-Blackwell.

Merino, Víctor (2017). «Victimización secundaria en los supuestos de violencia contra mujeres inmigrantes en situación administrativa irregular». Migraciones, 41, 107-131. <https://doi.org/10.14422/mig.i41.y2017.005>

Micolta, Amparo (2005). «Teorías y conceptos asociados al estudio de las migraciones internacionales». Trabajo Social, 7, 59-76.

Mohanty, Chandra (2008). «Bajo los ojos de Occidente: feminismo académico y discursos coloniales». En: SuÁrez, Liliana y Hernández, Rosalba (eds.). Descolonizando el feminismo. Teorías y prácticas desde los márgenes. Madrid: Cátedra.

Montero, Silvia y Rosario, Helia del (2015). «Diagnóstico: impacto de la crisis sobre las mujeres latinoamericanas en España». Disponible en:

<https://www.plataformaong.org/ARCHIVO/documentos/biblioteca/ 1457685818_dp177_b_red_investigacion_diagnostico_aieti.pdf >.

Monteros-Obelar, Silvina (2017). «La emergencia de "lo político" en el cruce entre migraciones femeninas, apoyo mutuo y participación política: la experiencia de la Red de Mujeres Latinoamericanas y del Caribe en España». Quaderns-e, 22 (2), 150-166.

Moriana, Gabriela (2018). «La violencia de género en las historias de vida de las mujeres inmigrantes». Aposta. Revista de Ciencias Sociales, 78, 97-120.

Muñoz-Arce, Gianinna (2018). "Contra la exclusión: lugar de enunciación e intervención social en la primera línea». Polis [en línea], 49. <http://journals.openedition.org/polis/15213>.

Muñoz de Bustillo, Rafael y Antón, José (2010). «De la España que emigra a la España que acoge: contexto, dimensiones y características de la inmigración latinoamericana en España». America Latina Hoy, 55, 15-39.

Nadler, Arie y Shnabel, Nurit (2015). «Intergroup reconciliation: instrumental and socio-emotional processes and the needs-based model». Eur Rev Soc Psycholgy, 26, 93-125. <https://doi.org/10.1080/10463283.2015.1106712>

Nijensohn, Melena y Minici, María (2017). «8M: articulación y resistencia frente a la precarización neoliberal. Una lectura de la experiencia asamblearia feminista en Argentina desde las coordenadas teóricas de J. Butler». Boletín Onteaiken, 24, 71-85. <http://onteaiken.com.ar/ver/boletin24/onteaiken24-07.pdf>.

Oso, Laura (2000). «Estrategias migratorias de las mujeres ecuatorianas y colombianas en situación irregular: servicio doméstico y prostitución en Galicia, Madrid y Pamplona». II Congreso de la Inmigración en España. Madrid

Oso, Laura y Parella, Sònia (2012). «Presentación. Inmigración, género y mercado de trabajo: una panorámica de la investigación sobre la inserción laboral de las mujeres inmigrantes en España». Cuaderno de Relaciones Laborales, 30(1), 11-44 
Parella, Sònia (2003). «La inserción laboral de la mujer inmigrante en los servicios de proximidad en Cataluña». Revista Internacional de Sociología (RIS), 36, 85-113. <https://doi.org/10.3989/ris.2003.i36.315>

- (2005). Segregación laboral y vulnerabilidad social de la mujer inmigrante a partir de la integración entre la clase social, el género y la etnia. Ministerio de Trabajo y Asuntos Sociales, Instituto de la Mujer.

Parella, Sònia y Petroff, Alisa (2014). «Migración de retorno en España: salidas de inmigrantes y programas de retorno en un contexto de crisis». CIDOB. Anuario de la Inmigración en España, 63-87.

Pedone, Claudia (2002). «El potencial del análisis de las cadenas y redes migratorias de las migraciones internacionales contemporáneas». Actas del III Congreso sobre la Inmigración en España. Contextos y Alternativas, 2, 223-235.

PÉrez, María (2008). «Mujeres inmigrantes: realidades, estereotipos y perspectivas educativas». Revista Española de Educación Comparada, 14, 137-175.

Pollack, Shoshana (2000). «Reconceptualizing Women’s Agency and Empowerment: Challenges to Self-Esteem Discourse and Women's Lawbreaking». Women \& Criminal Justice, 12 (1), 75-89. <https://doi.org/10.1300/J012v12n01_05>

Ray Vollhardt, Johanna; OKuyan, Mukadder y Ünal, Helin (2020). «Resistance to collective victimization and oppression». Current Opinion in Psychology, 35, 92-97. <https://doi.org/10.1016/j.copsyc.2020.04.001>

Salem, Rania; Cheong, Yuk; Miedema, Stephanie y Yount, Kathryn (2020). «Women's agency in Egypt: construction and validation of a multidimensional scale in rural Minya». Eastern Mediterranean Health Journal, 26 (6), 652-659. <https://doi.org/10.26719/2020.26.6.652>

SÁNCHEZ, Blanca (2011). «La política migratoria en España. Un análisis de largo plazo». Revista Internacional de Sociología, 69 (M1), 243-268. <https://doi.org/10.3989/ris.2011.iM1.393>

SASSEN, Saskia (2003). Contrageografías de la globalización. Género y ciudadanía en los circuitos transfronterizos. Madrid: Traficantes de Sueños.

SEN, Amartya (1985). «Well-being, agency, and freedom: the Dewey lectures 1984». The Journal of Philosophy, 82 (4), 69-221. <https://doi.org/10.2307/2026184>

Sipi, Remei (2000). «Las asociaciones de mujeres, ¿Agentes de integración social?». Papers, 60, 355-364. <https://doi.org/10.5565/rev/papers/v60n0.1048>

Sмiтh, Barbara (1983). Home Girls: A black feminist anthology. Nueva York: Table/ Women of Color Press.

SuÁrez, Liliana (2006). "Ciudadanía y migración: ¿un oxímoron?». Punto de Vista, 4, 29-48.

Subirats, Joan (2010). Ciudadanía e inclusión social. El tercer sector y las politicas públicas de acción social. Barcelona: Fundación Catalana L'Esplai.

Wade, Peter; Urrea, Fernando y Viveros, Mara (2008). «Identidades racializadas y sexualidades en América Latina. A manera de introducción». En: WADE, Peter; Urrea, Fernando y Viveros, Mara (eds.). Raza, etnicidad y sexualidades: ciudadanía y multiculturalismo en América Latina. Bogotá: Universidad Nacional de Colombia.

WiLLERS, Susanne (2016). «Migración y violencia: las experiencias de mujeres migrantes centroamericanas en tránsito por México». Sociológica, 31 (89), 163-195. 
